\section{Uso do Índice de Massa Corporal na avaliação do estado nutricional de adultos indígenas Xavánte, Terra Indígena Sangradouro-Volta Grande, Mato Grosso, Brasil}

\author{
Use of Body Mass Index to evaluate \\ the nutritional status of Xavánte Indigenous \\ adults, Sangradouro-Volta Grande, \\ Mato Grosso State, Central Brazil
}

Silvia Angela Gugelmin 1

Ricardo Ventura Santos 2,3

\section{Introdução}

This study evaluates the relationship between Body Mass Index (BMI) and anthropometric measurements in Xavánte adults on the Sangradouro-Volta Grande Indian Reservation, Mato Grosso State, Central Brazil. The objective was to analyze the use of BMI as an indicator of $n u$ tritional status in this specific population. Fieldwork was conducted in January-February 1999. Data include body mass, height, arm, hip, and waist circumference, and triceps skinfold of 128 adults over 20 years of age. The results indicate high correlations $(r>0.80)$ between BMI and body mass and waist and mid upper arm circumference for both sexes. For women, fat arm area and total arm area were also highly correlated with BMI, while for men hip circumference and total arm area showed a high correlation with BMI. The results suggest that high $B M I$ values are related to excess fat among the Xavánte. It is important to monitor changes in the nutritional status of indigenous peoples in Brazil, since there is evidence that such populations are undergoing rapid processes of nutritional transition.

South American Indians; Obesity; Nutritional Status; Anthropometry
A avaliação do estado nutricional de adultos é geralmente realizada por meio da análise da composição corporal, o que requer métodos que avaliem as reservas energéticas e a massa metabolicamente ativa 1,2,3. Existem várias técnicas para determinar a composição corporal, dentre elas as medidas antropométricas, que têm sido amplamente aplicadas. A coleta dessas medidas envolve baixo custo operacional e apresenta relativa simplicidade na sua aplicação, particularmente as medidas de massa corporal e estatura 4 . Para estimar a composição corporal, várias relações entre estatura e massa corporal têm sido desenvolvidas, como, por exemplo, o índice de Quételet, que preenche o requisito de baixa correlação com a estatura e correlações mais elevadas com a massa corporal e medidas de gordura corporal 1 . O índice de Quételet, também denominado Índice de Massa Corporal (IMC), é calculado pela razão entre massa corporal (kg) e estatura (m) ao quadrado, sendo amplamente empregado em estudos epidemiológicos.

Embora o IMC não meça diretamente a proporção de gordura corporal e não defina a distribuição desta, estudos realizados em grandes amostras populacionais, basicamente européias e norte-americanas, têm revelado alta correlação entre IMC e gordura corporal e, mais importante, aumento do risco de mortalidade as- 
sociado a altos valores de IMC 3,5. Este índice parece ser adequado para expressar a gordura corporal total em estudos populacionais 6 , considerando-se que valores iguais ou superiores a $30 \mathrm{~kg} \cdot \mathrm{m}-2$ em adultos indicam indivíduos obesos 3 .

A literatura sobre avaliação nutricional de adultos com base no IMC considera, sobretudo, a análise de dados antropométricos oriundos de populações predominantemente de origem européia e norte-americana 1,3. Estudos realizados em populações aborígines na Austrália e polinésias têm demonstrado importantes efeitos da etnia na proporção e composição corporal, com conseqüências em potencial sobre o IMC 4,7. A distribuição de gordura, a estatura sentada, a densidade óssea e a massa muscular contribuem para as diferenças no perfil de IMC encontradas entre as mulheres polinésias e européias da Nova Zelândia, determinando que valores de IMC entre 25 e $30 \mathrm{~kg} . \mathrm{m}^{-2}$ possam ser considerados normais entre as primeiras 8 . Por outro lado, segundo Norgan 4, para os grupos aborígines que vivem na Austrália, valores abaixo de 18,5kg.m-2 não necessariamente indicam baixo peso, mas uma proporção corporal diferenciada daquelas reportadas para populações européias. Eveleth \& Tanner 9 evidenciam que o comprimento das pernas em relação à estatura sentada é maior entre os povos aborígines e africanos que nas populações européias, americanas e asiáticas, o que pode influenciar os valores do IMC.

Em relação às populações indígenas no Brasil, estudos antropométricos têm utilizado o IMC para analisar o perfil nutricional, inclusive na caracterização de sobrepeso e obesidade 10 , $11,12,13,14$. No entanto, nenhum discutiu a adequação desse índice na avaliação do estado nutricional das populações indígenas. Em face do exposto, o presente artigo tem como objetivo comparar valores de IMC com medidas mais diretas de composição corporal de uma amostra de adultos Xavánte, a fim de averiguar a potencialidade do IMC como instrumento de avaliação do estado nutricional na caracterização do sobrepeso e obesidade.

\section{População e métodos}

A presente pesquisa foi desenvolvida na aldeia São José, localizada na Terra Indígena (TI) Sangradouro-Volta Grande, no leste do Estado do Mato Grosso, entre $15^{\circ} 11^{\prime}$ de latitude Sul e 5335' de longitude Oeste. A TI está estabelecida numa área adscrita aos municípios General Carneiro, Novo São Joaquim e Poxoréo. Em 1997, a população total era de 920 Xavánte, pertencen- tes ao tronco lingüístico Macro-Jê e distribuídos em sete aldeias 15 .

O trabalho de campo, realizado durante os meses de janeiro e fevereiro de 1999, envolveu a coleta de dados antropométricos de toda a população presente na aldeia. Esse trabalho faz parte de um estudo mais amplo desenvolvido na comunidade durante 1998/1999, quando foram coletados dados a respeito das condições de saneamento, alocação de tempo, crescimento físico e morbidade referida ${ }^{13}$. A pesquisa foi aprovada pela Comissão Nacional de Ética em Pesquisa (CONEP), Fundação Nacional do Índio (FUNAI) e pela população residente na aldeia.

O censo realizado no início do trabalho de campo revelou uma população total de 590 indivíduos, dos quais 192 apresentavam idade de vinte anos ou mais. Para esta análise, foram excluídas as mulheres grávidas $(n=10)$ e algumas pessoas que haviam migrado recentemente para outras aldeias $(n=9)$. As perdas incluíram indivíduos que estavam ausentes no momento da coleta dos dados antropométricos $(n=19)$. Além disso, 26 indivíduos se recusaram a participar do estudo. Portanto, a amostra final ficou em 128 indivíduos adultos.

As medidas antropométricas avaliadas foram a massa corporal (MC), estatura (EST), perímetro braquial (PB), do quadril (PQUA) e do abdômen (PABD), além da dobra cutânea tricipital (DCT). As medições foram efetuadas nas casas dos indivíduos. Foram utilizados os seguintes instrumentos: balança mecânica de plataforma marca Seca (modelo 761, Hamburgo, Alemanha), com capacidade máxima de $150 \mathrm{~kg}$ e resolução de 0,5kg; antropômetro metálico marca GPM (Zurique, Suíça), com resolução de $1 \mathrm{~mm}$; adipômetro marca Lange (Cambridge, Estados Unidos), com resolução de $1 \mathrm{~mm}$; e fita métrica flexível com resolução de $1 \mathrm{~mm}$. O PB foi medido no ponto médio do braço direito, entre o processo acrômico do ombro e o fim do olecrâneo no cotovelo. A DCT foi aferida três vezes, sobre o tríceps, na intercessão entre o ponto médio do braço direito e a linha posterior do braço. A medição do PABD foi efetuada na altura da cintura, sobre a cicatriz umbilical, enquanto o PQUA foi medido na altura da maior extensão das nádegas. Os indivíduos foram medidos com roupas leves e descalços, seguindo as técnicas recomendadas por Lohman et al. 16 .

Foram construídos índices com base nos dados antropométricos, incluindo o IMC; a razão cintura-quadril (RCQ), definida como a razão entre o perímetro da cintura e o perímetro do quadril; a área muscular e a área gordurosa do braço, calculadas de acordo com as fórmulas de Frisancho modificadas por Heymsfield et al. 17. 
Os indivíduos foram classificados segundo o IMC utilizando-se os pontos de corte recomendados pela Organização Mundial da Saúde (OMS) 3: IMC $\leq 18,4 \mathrm{~kg} \cdot \mathrm{m}^{-2}$ - baixo peso; 18,5 a $24,9 \mathrm{~kg} \cdot \mathrm{m}^{-2}$ - adequado; 25,0 a $29,9 \mathrm{~kg} \cdot \mathrm{m}^{-2}$ - sobrepeso; e valores de IMC $\geq 30,0 \mathrm{~kg} \cdot \mathrm{m}^{-2}$ - obesidade.

As análises estatísticas foram realizadas no programa SPSS (SPSS Inc., Chicago, Estados Unidos) e Epi Info 6.01 (Centers for Disease Control and Prevention, Atlanta, Estados Unidos). Foram calculadas as médias e desvios padrão para todas as medidas antropométricas segundo sexo e faixa etária, bem como os valores de correlação de Pearson. As comparações de variáveis contínuas foram realizadas por meio do teste $t$ Student.

\section{Resultados}

A participação no estudo antropométrico foi de aproximadamente $67 \%$ dos adultos presentes em São José em janeiro e fevereiro de 1999. Tratava-se de uma população com média de idade de 36,8 anos para homens e 34,7 anos para mulheres ( $t$ Student $=0,86 ; \mathrm{p}=0,3913$ ). A amostra estudada não apresentou diferenças na distribuição por sexo $(49,2 \%$ eram mulheres e 50,8\% homens).

No total de indivíduos examinados, observou-se que $78 \%$ apresentavam excesso de massa corporal a partir dos pontos de corte de IMC propostos pela OMS 3 (Figura 1). A freqüência de sobrepeso e obesidade foi maior entre as mulheres $(90,5 \%)$.

A Tabela 1 descreve os valores médios e desvios padrão das medidas antropométricas. Os homens apresentam médias de estatura, massa corporal e área muscular do braço mais elevadas que as mulheres em todas as faixas etárias. Para as outras medidas, isto é, perímetro braquial, dobra cutânea tricipital, perímetro abdominal, perímetro do quadril, índice de massa corporal e área de gordura do braço, os valores médios foram mais altos entre as mulheres.

A distribuição das categorias de IMC segundo faixas etárias é apresentada na Tabela 2 . Evidenciou-se maior ocorrência de sobrepeso e obesidade nas mulheres em todas as faixas etárias. Todas as mulheres entre 40 a 59,9 anos apresentaram excesso de peso. No caso dos homens, o sobrepeso foi encontrado em todas as faixas de idade, enquanto a obesidade concentrou-se entre 30 e 49,9 anos.

Para os homens (Tabela 3), verificaram-se altas correlações positivas do IMC com a massa corporal $(\mathrm{r}=0,906 ; \mathrm{p}=0,000)$, o perímetro abdominal $(\mathrm{r}=0,889 ; \mathrm{p}=0,000)$, o perímetro do quadril ( $r=0,888 ; p=0,000)$, o perímetro braquial $(\mathrm{r}=0,875 ; \mathrm{p}=0,000)$, área muscular do braço $(\mathrm{r}=0,773 ; \mathrm{p}=0,000)$ e a área total do braço $(\mathrm{r}=0,873 ; \mathrm{p}=0,000)$. O IMC correlacionou-se positivamente, mas com valores menores, com a área de gordura do braço $(r=0,658 ; \mathrm{p}=0,000)$, com a dobra tricipital $(r=0,564 ; p=0,000)$ e com

Figura 1

Distribuição relativa das categorias do Índice de Massa Corporal (IMC) da população adulta Xavánte acima de vinte anos, segundo sexo. Aldeia São José, Terra Indígena Sangradouro-Volta Grande, Mato Grosso, Brasil, 1998/1999.

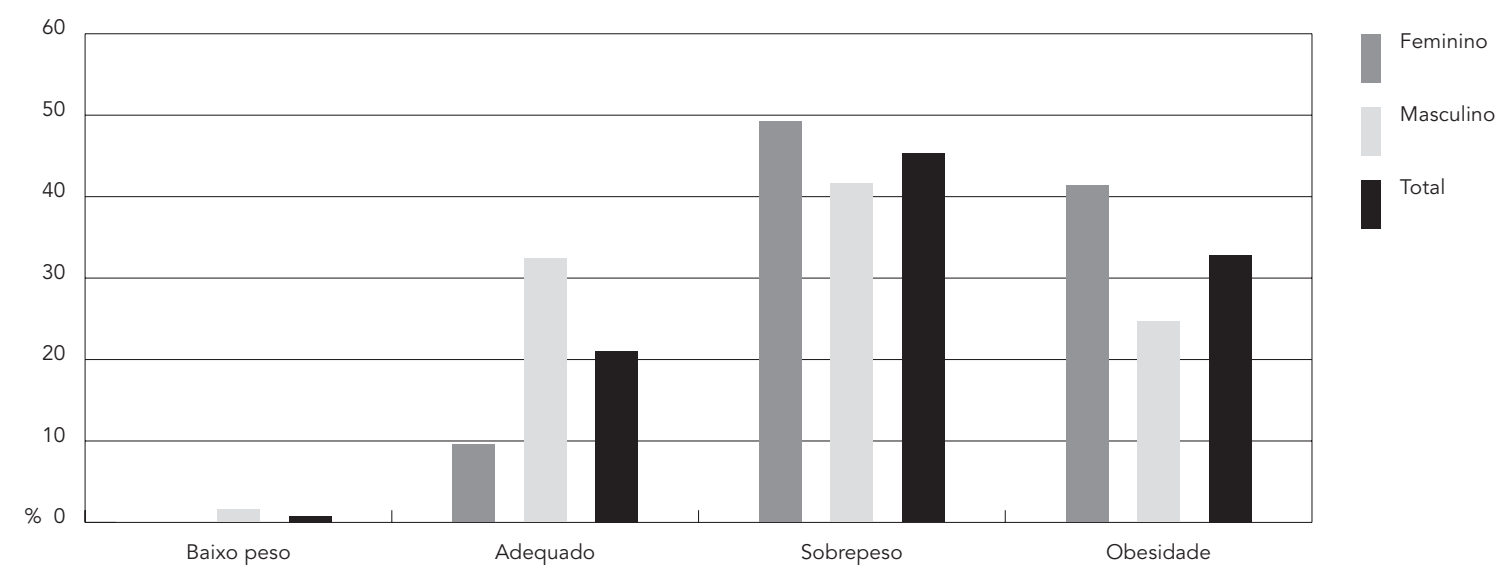


Tabela 1

Médias e desvios-padrão das medidas antropométricas de adultos Xavánte ( $\geq 20$ anos), segundo sexo.

Aldeia São José, Terra Indígena Sangradouro-Volta Grande, Mato Grosso, Brasil, 1998/1999.

\begin{tabular}{|c|c|c|c|c|c|c|c|c|c|c|c|c|c|c|c|c|c|c|c|}
\hline \multirow{2}{*}{$\begin{array}{l}\text { Faixa etária } \\
\text { (anos) }\end{array}$} & \multirow[t]{2}{*}{$\mathrm{n}$} & \multicolumn{2}{|c|}{$\mathrm{EST}(\mathrm{cm})$} & \multicolumn{2}{|c|}{$M C(k g)$} & \multicolumn{2}{|c|}{ IMC (kg.m-2) } & \multicolumn{2}{|c|}{ PB $(\mathrm{cm})$} & \multicolumn{2}{|c|}{ DCT $(\mathrm{mm})$} & \multicolumn{2}{|c|}{ PABD $(\mathrm{cm})$} & \multicolumn{2}{|c|}{ PQUA $(\mathrm{cm})$} & \multicolumn{2}{|c|}{ AGB $(\mathrm{cm})$} & \multicolumn{2}{|c|}{ AMB $(\mathrm{cm})$} \\
\hline & & $x$ & $\mathrm{DP}$ & $x$ & DP & $x$ & $\mathrm{DP}$ & $x$ & $\mathrm{DP}$ & $x$ & $\mathrm{DP}$ & $x$ & $\mathrm{DP}$ & $x$ & DP & $x$ & DP & $x$ & DP \\
\hline \multicolumn{20}{|l|}{ Masculino } \\
\hline $20,0-29,9$ & 29 & 167,5 & 6,2 & 71,5 & 10,7 & 25,4 & 3,2 & $30,8^{*}$ & 2,9 & 7,6 & 3,0 & $86,6^{*}$ & 9,5 & $98,2^{*}$ & 7,0 & $11,5^{\star}$ & 5,3 & $64,9^{*}$ & 11,1 \\
\hline $30,0-39,9$ & 17 & 166,4 & 5,6 & 82,9 & 12,0 & 29,9 & 3,6 & $33,1^{*}$ & 3,0 & $10,2^{*}$ & 4,8 & $97,7^{\star}$ & 8,4 & $104,7^{*}$ & 5,8 & $16,2^{*}$ & 8,0 & $71,9^{*}$ & 11,7 \\
\hline $40,0-49,9$ & 7 & 165,7 & 4,6 & 79,1 & 10,5 & 28,8 & 3,1 & $32,2^{*}$ & 2,4 & $11,6^{*}$ & 3,8 & $101,1^{*}$ & 3,6 & 103,0 * & 4,8 & $17,5^{\star}$ & 5,8 & $65,4^{*}$ & 11,1 \\
\hline $50,0-59,9$ & 6 & 163,1 & 4,2 & 75,1 & 11,4 & 28,2 & 3,5 & $32,3^{*}$ & 2,2 & 9,1 * & 2,0 & $96,7^{*}$ & 9,2 & $98,8^{*}$ & 5,1 & $14,1^{*}$ & 3,3 & $69,4^{*}$ & 10,7 \\
\hline 60,0 e mais & 6 & 170,1 & 4,8 & 70,3 & 7,0 & 24,4 & 2,8 & 29,9 & 1,3 & 8,6 & 2,7 & 90,4 & 6,2 & 97,6 & 2,9 & 12,3 & 4,0 & 58,9 & 4,3 \\
\hline Total & 65 & 166,8 & 5,7 & 75,5 & 11,7 & 27,1 & 3,8 & 31,6 & 2,9 & 8,9 & 3,7 & 92,2 & 10,0 & 100,4 & 6,6 & 13,7 & 6,3 & 66,6 & 11,1 \\
\hline \multicolumn{20}{|l|}{ Feminino } \\
\hline $20,0-29,9$ & 26 & 155,2 & 4,3 & 71,4 & 11,9 & 29,6 & 4,4 & 32,5 & 3,0 & 19,4 & 5,0 & 101,0 & 13,3 & $107,5^{\star}$ & 15,2 & 28,9 & 8,8 & 56,1 & 9,5 \\
\hline $30,0-39,9$ & 23 & 155,2 & 2,4 & 73,9 & 12,5 & 30,6 & 4,7 & 33,5 & 3,3 & 21,3 & 5,4 & 109,1 & 14,3 & 108,1 & 8,7 & 32,5 & 10,4 & 57,5 & 10,2 \\
\hline $40,0-49,9$ & 6 & 155,7 & 3,5 & 68,8 & 9,4 & 28,4 & 4,0 & $33,5^{\star}$ & 2,8 & $19,1^{*}$ & 5,0 & $102,1^{*}$ & 10,3 & $104,9 *$ & 6,2 & $29,1^{*}$ & 8,3 & $60,6^{*}$ & 12,8 \\
\hline $50,0-59,9$ & 3 & 153,5 & 2,8 & 64,7 & 6,8 & 27,5 & 3,1 & 33,8 & 4,0 & 14,8 & 6,2 & 104,0 & 2,6 & 101,3 & 8,1 & 23,7 & 11,8 & 67,9 & 13,0 \\
\hline 60,0 e mais & 5 & 156,2 & 4,7 & 70,0 & 13,2 & 28,5 & 4,0 & 32,9 & 2,2 & 17,8 & 3,7 & 106,5 & 9,0 & 106,1 & 8,3 & 26,9 & 6,7 & 59,3 & 6,4 \\
\hline Total & 63 & 155,3 & 3,5 & 71,6 & 11,8 & 29,6 & 4,4 & 33,1 & 3,0 & 19,7 & 5,2 & 104,7 & 13,1 & 107,1 & 11,5 & 29,8 & 9,4 & 57,8 & 10,0 \\
\hline
\end{tabular}

* O número de indivíduos avaliados nestas categorias diferem da amostra geral em menos um ou menos dois indivíduos.

$\mathrm{EST}=$ estatura; $\mathrm{MC}=$ massa corporal; IMC = Índice de Massa Corporal; PB = perímetro braquial;

$\mathrm{DCT}$ = dobra cutânea tricipital; PABD = perímetro abdominal; PQUA = perímetro do quadril;

$\mathrm{AGB}=$ área de gordura do braço; $\mathrm{AMB}=$ área muscular do braço.

Tabela 2

Distribuição absoluta e relativa das categorias de Índice de Massa Corporal (IMC) para a população adulta ( $\geq 20$ anos), segundo faixa etária e sexo. Aldeia São José, Terra Indígena Sangradouro-Volta Grande, Mato Grosso, Brasil, 1998/1999.

\begin{tabular}{|c|c|c|c|c|}
\hline \multirow{2}{*}{$\begin{array}{l}\text { Faixa etária } \\
\text { (anos) }\end{array}$} & \multicolumn{4}{|c|}{ IMC } \\
\hline & $\begin{array}{c}\text { Baixo peso } \\
n(\%)\end{array}$ & $\begin{array}{c}\text { Adequado } \\
n(\%)\end{array}$ & $\begin{array}{c}\text { Sobrepeso } \\
\text { n (\%) }\end{array}$ & $\begin{array}{c}\text { Obeso } \\
\text { n (\%) }\end{array}$ \\
\hline \multicolumn{5}{|l|}{ Masculino } \\
\hline $20-29,9$ & $1(3,4)$ & $14(48,4)$ & $11(37,9)$ & $3(10,3)$ \\
\hline $30-39,9$ & - & $2(11,8)$ & $6(35,3)$ & $9(52,9)$ \\
\hline $40-49,9$ & - & $1(14,2)$ & $3(42,9)$ & $3(42,9)$ \\
\hline $50-59,9$ & - & $1(16,7)$ & $4(66,6)$ & $1(16,7)$ \\
\hline 60 e mais & - & $3(50,0)$ & $3(50,0)$ & - \\
\hline Total & $1(1,5)$ & $21(32,3)$ & $27(41,6)$ & $16(24,6)$ \\
\hline \multicolumn{5}{|l|}{ Feminino } \\
\hline $20-29,9$ & - & $3(11,5)$ & $13(50,0)$ & $10(38,5)$ \\
\hline $30-39,9$ & - & $1(4,3)$ & $10(43,5)$ & $12(52,2)$ \\
\hline $40-49,9$ & - & - & $5(83,3)$ & $1(16,7)$ \\
\hline $50-59,9$ & - & - & $2(66,7)$ & $1(33,3)$ \\
\hline 60 e mais & - & $2(40,0)$ & $1(20,0)$ & $2(40,0)$ \\
\hline Total & - & $6(9,5)$ & $31(49,2)$ & $26(41,3)$ \\
\hline
\end{tabular}

a razão cintura-quadril $(r=0,628 ; \mathrm{p}=0,000)$. Detectou-se baixa correlação do IMC com a estatura $(r=0,075 ; \mathrm{p}=0,574)$.

Entre as mulheres (Tabela 3), o IMC apresentou altas correlações com a massa corporal $(\mathrm{r}=0,965 ; \mathrm{p}=0,000)$ e com as medidas de composição corporal, isto é, dobra do tríceps ( $\mathrm{r}=$ $0,728 ; \mathrm{p}=0,000)$, área de gordura do braço $(\mathrm{r}=$ $0,816 ; p=0,000)$, perímetro braquial $(r=0,821$; $\mathrm{p}=0,000)$, perímetro abdominal $(\mathrm{r}=0,807 ; \mathrm{p}=$ $0,000)$ e área total do braço $(r=0,822 ; p=0,000)$. Os valores da correlação com a área muscular do braço $(r=0,567 ; p=0,000)$ e com a razão cintura-quadril ( $r=0,346 ; p=0,009)$ foram menores. Da mesma forma que, para os homens, observou-se baixa correlação com a estatura ( $\mathrm{r}=$ $0,194 ; \mathrm{p}=0,145)$.

\section{Discussão}

A obesidade, uma doença prevenível que acarreta diversas enfermidades associadas, é relativamente comum na maior parte dos países industrializados e está aumentando nos países em desenvolvimento 3,18 .

O emprego do IMC como indicador do estado nutricional de adultos tem sido amplamente discutido, principalmente no que se re- 
Matriz de correlação das variáveis antropométricas de adultos Xavánte ( $\geq 20$ anos), segundo sexo.

Aldeia São José, Terra Indígena Sangradouro-Volta Grande, Mato Grosso, Brasil, 1998/1999.

\begin{tabular}{|c|c|c|c|c|c|c|c|c|c|c|}
\hline Variáveis & $M C(k g)$ & EST $(\mathrm{cm})$ & IMC (kg.m-2) & DCT (mm) & PB (cm) & PABD $(\mathrm{cm})$ & PQUA (cm) & AMB $(\mathrm{cm})$ & AGB $(\mathrm{cm})$ & $\mathrm{RCQ}(\mathrm{cm})$ \\
\hline \multicolumn{11}{|l|}{ Masculino } \\
\hline Estatura & $0,485^{\star \star}$ & - & & & & & & & & \\
\hline IMC & $0,906^{\star \star}$ & $0,075(\mathrm{~ns})$ & - & & & & & & & \\
\hline DCT & $0,654^{\star \star}$ & $0,369 * \star$ & $0,564^{\star \star}$ & - & & & & & & \\
\hline PB & $0,872^{\star \star}$ & $0,284^{\star}$ & $0,875^{\star \star}$ & $0,581^{\star \star}$ & - & & & & & \\
\hline PABD & $0,889 * \star$ & $0,292^{*}$ & $0,889 * \star$ & $0,672^{\star \star}$ & $0,792^{\star \star}$ & - & & & & \\
\hline PQUA & $0,926^{* *}$ & $0,403^{\star *}$ & $0,888^{\star *}$ & $0,606^{* *}$ & $0,817^{\star \star}$ & $0,866^{* *}$ & - & & & \\
\hline AMB & $0,718^{\star \star}$ & $0,141(n s)$ & $0,773^{\star \star}$ & $0,201(n s)$ & $0,913^{\star *}$ & $0,615^{\star \star}$ & $0,674^{\star \star}$ & - & & \\
\hline$A G B$ & $0,742^{\star \star}$ & $0,385^{\star \star}$ & $0,658^{\star *}$ & $0,985^{\star \star}$ & $0,701^{\star \star}$ & $0,728^{\star \star}$ & $0,684^{\star \star}$ & $0,354^{\star \star}$ & - & \\
\hline $\mathrm{RCO}$ & $0,588^{\star \star}$ & $0,091(n s)$ & $0,628^{\star \star}$ & $0,547^{\star \star}$ & $0,534^{\star \star}$ & $0,850^{\star \star}$ & $0,473^{\star \star}$ & $0,371^{\star \star}$ & $0,561^{\star \star}$ & - \\
\hline Área & $0,868^{\star \star}$ & $0,274^{*}$ & $0,873^{\star \star}$ & $0,580^{\star \star}$ & $0,998^{\star \star}$ & $0,784^{\star \star}$ & $0,809 * \star$ & $0,914^{\star *}$ & $0,703^{* \star}$ & $0,525^{\star *}$ \\
\hline \multicolumn{11}{|l|}{ Feminino } \\
\hline Estatura & $0,440^{\star \star}$ & - & & & & & & & & \\
\hline IMC & $0,965^{\star \star}$ & $0,194(n s)$ & - & & & & & & & \\
\hline DCT & $0,726^{\star \star}$ & $0,218(n s)$ & $0,728^{\star \star}$ & - & & & & & & \\
\hline PB & $0,794^{\star \star}$ & $0,159(n s)$ & $0,821^{\star \star}$ & $0,659^{\star *}$ & - & & & & & \\
\hline PABD & $0,830^{\star \star}$ & $0,355^{\star \star}$ & $0,807^{* *}$ & $0,601^{* \star}$ & $0,623^{\star *}$ & - & & & & \\
\hline PQUA & $0,726^{\star \star}$ & $0,310^{*}$ & $0,701^{\star \star}$ & $0,512^{\star \star}$ & $0,451^{\star \star}$ & $0,688^{\star \star}$ & - & & & \\
\hline AMB & $0,532^{\star \star}$ & $0,055(\mathrm{~ns})$ & $0,567^{* *}$ & 0,159 (ns) & $0,846^{\star \star}$ & $0,395^{\star \star}$ & 0,233 (ns) & - & & \\
\hline$A G B$ & $0,810^{\star *}$ & $0,223(\mathrm{~ns})$ & $0,816^{* *}$ & $0,966^{* *}$ & $0,820 * *$ & $0,648^{* *}$ & $0,531^{\star \star}$ & $0,394^{\star *}$ & - & \\
\hline $\mathrm{RCO}$ & $0,353^{\star *}$ & $0,166(\mathrm{~ns})$ & $0,346^{* *}$ & $0,278^{*}$ & $0,357^{\star \star}$ & $0,650^{\star \star}$ & $-0,091$ (ns) & $0,271^{*}$ & $0,311^{*}$ & - \\
\hline Área & $0,797^{\star \star}$ & $0,163(\mathrm{~ns})$ & $0,822^{\star *}$ & $0,656^{\star *}$ & $0,998^{\star \star}$ & $0,619 * *$ & $0,451^{\star \star}$ & $0,848^{* *}$ & $0,821^{\star \star}$ & $0,347^{* *}$ \\
\hline
\end{tabular}

$* 0,05>p>0,01$;

$* * p<0,01$;

ns $=$ não significativo

$\mathrm{MC}=$ massa corporal; $\mathrm{EST}$ = estatura; $\mathrm{IMC}=$ Índice de Massa Corporal; $\mathrm{DCT}=$ dobra cutânea tricipital;

$\mathrm{PB}=$ perímetro braquial; $\mathrm{PABD}$ = perímetro abdominal; $\mathrm{PQUA}=$ perímetro do quadril; $\mathrm{AMB}=$ área muscular do braço:

$\mathrm{AGB}=$ área de gordura do braço; $\mathrm{RCQ}$ = razão cintura-quadril; Ârea = área total do braço.

fere à sua utilização na análise do sobrepeso e da obesidade 1,3,6,18. As críticas estão baseadas no fato de esse índice não distinguir a massa magra do tecido adiposo. Segundo Anjos 1, o fato de o IMC apresentar alta correlação com a massa corporal (normalmente valores superiores a 0,80 ) e baixa correlação com a estatura (r em torno de 0,10) não é suficiente. É importante que os valores de IMC se correlacionem com outras medidas, como percentual de gordura corporal, dobras cutâneas e perímetros. O autor reportou, em sua revisão da literatura, valores de correlação entre o IMC e a dobra cutânea tricipital acima de 0,60 ; o perímetro abdominal encontrado foi superior a 0,80 para diversas amostras norte-americanas e européias.

$\mathrm{O}$ inquérito antropométrico realizado com os adultos Xavánte de São José evidenciou valores de correlação entre o IMC e as medidas antropométricas que não se distanciam daque- les reportados por Anjos 1. Os achados indicaram uma alta correlação do IMC com massa corporal, perímetro abdominal, dobra cutânea tricipital, área total do braço e área de gordura do braço, para as mulheres Xavánte, e massa corporal, área total e muscular do braço e perímetros, para os homens. Segundo Garrow 6 e Malina 19 , as medidas de perímetro abdominal, dobra cutânea do tríceps e área de gordura do braço guardam relação direta com a quantidade de gordura total do corpo.

Ainda que o IMC, nas mais diversas populações, apresente uma alta correlação com medidas antropométricas de composição corporal, persiste a dificuldade em definir, para populações específicas, pontos de corte a fim de determinar o estado nutricional, incluindo baixo peso, normalidade, sobrepeso e obesidade. A utilização do IMC para classificar indivíduos de acordo com a adiposidade pode resultar em 
erros decorrentes da contribuição da idade no aumento da deposição de gordura; das diferentes contribuições da massa muscular, massa óssea e fluidos corporais no peso corpóreo e em virtude da influência das proporções corporais 1,18. Rush et al. 8 demonstraram que o ponto de corte recomendado pela OMS para definir sobrepeso (IMC de 25-30) não se aplica às mulheres polinésias. Um IMC menor ou igual a $30 \mathrm{~kg} \cdot \mathrm{m}^{-2}$ pode ser considerado normal para elas. Os autores relacionaram os achados às diferenças na distribuição da gordura, estatura sentada e massa muscular associadas às etnias. Por sua vez, Norgan 7 observou baixos valores de IMC em aborígines australianos. Aproximadamente $25 \%$ dos homens e $30 \%$ das mulheres apresentavam IMC entre 16 e 18,5kg.m-2, apesar de os valores de dobra cutânea subescapular apresentarem-se entre os percentis 2550 da população de referência norte-americana. Segundo o autor, esses valores implicam alta adiposidade subcutânea, enquanto os baixos valores de IMC podem estar associados, em parte, à baixa relação estatura sentada/estatura dos aborígines, por causa, principalmente, de suas longas pernas. Sendo assim, a proporcionalidade entre as dimensões corporais também parece afetar o IMC e, conseqüentemente, sua interpretação. Outros autores, como Malina 19 e Van Loan 20, sugeriram que existem grandes variações na massa magra (principalmente osso) e na distribuição da gordura corporal, relacionadas a idade, sexo, ambiente e etnia.

Desde 1990, a OMS 3,21 tem recomendado a utilização do IMC para avaliação do estado nutricional de adultos, sugerindo como limite máximo para a normalidade o valor de $25 \mathrm{~kg} . \mathrm{m}^{-2}$. No caso dos homens Xavánte, os valores médios de IMC estiveram acima de 25 nas faixas etárias de 30 a 59 anos. Para as mulheres, em todas as faixas etárias, os valores de IMC estiveram muito acima do limite de normalidade proposto. Dessa forma, de acordo com as recomendações da OMS, $80 \%$ da população adulta Xavánte de São José apresentou excesso de massa corporal.

Esses achados, sobretudo pela sua magnitude, podem indicar que (i) os pontos de corte de IMC empregados não são adequados para os Xavánte ou (ii) há um processo de transição nutricional em curso na população que está resultando em uma porcentagem bastante expressiva de indivíduos com sobrepeso e obesidade. A segunda possibilidade é particularmente plausível, a se julgar pelos resultados do presente estudo e de pesquisas anteriores realizadas entre os Xavánte 11,13,22. Com base em dados antropométricos coligidos por Neel et al. 23 entre os Xavánte de Pimentel Barbosa, na década de 1960, Coimbra Jr. et al. 22 demonstraram que a quase totalidade dos adultos (88\% ou 22 indivíduos num total de 25) apresentavam IMC na faixa de $18,5-24,9 \mathrm{~kg} \cdot \mathrm{m}^{-2}$. Somente três homens adultos jovens apresentavam valores de IMC acima de 25kg.m-2. Logo, no período em que os Xavánte tinham sua subsistência baseada em atividades de caça, pesca e coleta, quando era intensa a atividade física, aproximadamente $90 \%$ dos adultos apresentavam valores de IMC dentro da faixa de normalidade. Em 1990, quando a população já apresentava intensas mudanças em seu padrão de dieta e mobilidade, com a incorporação de alimentos industrializados e redução da atividade física, a distribuição dos valores do IMC mostrou maior proporção de indivíduos de ambos os sexos nas categorias de sobrepeso e obesidade (48,4\% e 4,4\%, respectivamente) 22 .

Em estudo que comparou a população Xavánte de Sangradouro-Volta Grande com a residente em Pimentel Barbosa, Gugelmin \& Santos 13 mostraram diferenças importantes nas prevalências de obesidade em adultos $(32,8 \%$ na aldeia de São José em Sangradouro-Volta Grande e 3,7\% na aldeia de Etéñitépa em Pimentel Barbosa). Os autores associaram esses resultados a diferentes padrões de atividade física e de consumo de alimentos, dentre outros determinantes.

Conforme anteriormente apontado por Gugelmin \& Santos 13, cuja pesquisa em São José já havia detectado elevadas prevalências de sobrepeso e obesidade em adultos, são ainda necessários estudos com vistas a caracterizar, em detalhes, os determinantes do excesso de peso evidenciados nessa comunidade. É preciso incorporar em pesquisas futuras a coleta de informações diacrônicas sobre estilo de vida, consumo alimentar, nível de atividade física, entre outras. Possivelmente, dificuldades na sustentabilidade das atividades de subsistência em face do aumento populacional, da restrição territorial e da intensificação do uso dos solos e dos recursos naturais, bem como mudanças no padrão de atividade física, a facilidade de acesso a centros urbanos e o recebimento de salários e aposentadorias trouxeram mudanças nas práticas alimentares e no estilo de vida do grupo, repercutindo sobre a composição corporal 11,13.

No caso do Brasil, ainda se têm poucos registros sobre a situação nutricional das populações indígenas 24,25,26. Alguns estudos têm apontado para elevadas freqüências de adultos com valores de IMC que, seguindo-se recomen- 
dações internacionais 3 , seriam indicativas da ocorrência disseminada de sobrepeso e obesidade $10,11,12,13,14$. No contexto de aceleradas mudanças sócio-econômicas e ambientais experimentadas pelas populações indígenas, torna-se importante a monitoração contínua do perfil nutricional, uma vez que altos valores de IMC e perímetro abdominal têm sido descritos como fatores de risco para doenças crônicas não transmissíveis, como a hipertensão e o diabetes 27 .

\section{Resumo}

Este artigo examina as relações entre o Índice de Massa Corporal (IMC) e medidas antropométricas de composição corporal de adultos Xavánte, da aldeia São José, Terra Indígena Sangradouro-Volta Grande, Mato Grosso, Brasil, a fim de analisar a potencialidade do IMC como instrumento de avaliação do estado nutricional desse grupo indígena. O trabalho de campo foi realizado em janeiro e fevereiro de 1999, sendo aferidas medidas de massa corporal (MC); estatura (EST); perímetro braquial (PB), do quadril (PQUA) e do abdômen (PABD), e dobra cutânea tricipital (DCT) de 128 indivíduos. Os achados indicam, para ambos os sexos, uma alta correlação $(r>0,80)$ dos valores de IMC com a MC, PB e PABD. Houve, ainda, alta correlação do IMC com as áreas total e de gordura do braço, para as mulheres, e com o PQUA e a área total do braço, para os homens. Esses resultados sugerem que altos valores de IMC indicam excesso de tecido adiposo entre os Xavánte. Diante das evidências de um intenso processo de transição nutricional, resultando em aumento nas freqüencias de sobrepeso e obesidade em adultos, é importante monitorar o perfil nutricional dos povos indígenas no Brasil.

Índios Sul-Americanos; Obesidade; Estado Nutricional; Antropometria
Portanto, apesar de o índice de massa corporal não indicar diretamente a composição corporal do indivíduo, parece válida a sua utilização na avaliação do estado nutricional de adultos Xavánte em virtude da alta correlação com as medidas antropométricas indicativas de acúmulo de gordura corporal. Além disso, é fundamental o uso de índices simples e de fácil incorporação na rotina das ações de saúde, destacando-se o IMC, especialmente como indicador de risco para a obesidade e as doenças cardiovasculares em jovens adultos.

\section{Colaboradores}

S. A. Gugelmin realizou a coleta de dados, contribuiu na idéia original do artigo, na análise dos dados e na elaboração da primeira versão do artigo. R. V. Santos contribuiu na análise dos dados e na revisão crítica do artigo.

\section{Agradecimentos}

Esta pesquisa foi financiada pelo Centro Nacional de Epidemiologia-Sistema Nacional de Vigilância em Saúde (CENEPI-VIGISUS) e pelo Programa de Apoio à Pesquisa Estratégica em Saúde, Fundação Oswaldo Cruz (PAPES/FIOCRUZ). Agradecemos a Inês Rugani Ribeiro de Castro pelas valiosas sugestões e aos Xavánte de São José, que nos acolheram com tanto carinho. 


\section{Referências}

1. Anjos LA. Índice de massa corporal (massa corporal.estatura-2) como indicador do estado nutricional de adultos: revisão de literatura. Rev Saúde Pública 1992; 26:431-6.

2. Monteiro JC. Obesidade: diagnóstico, métodos e fundamentos. In: Halpern A, Matos AFG, Suplicy HL, Mancini MC, Zanella MT, organizadores. Obesidade. São Paulo: Lemos Editoral; 1998. p. 31-53.

3. World Health Organization. Obesity. Preventing and managing the global epidemic. Geneva: World Health Organization; 1998. (Report of WHO Consultation on Obesity).

4. Norgan NG. Population differences in body composition in relation to the body mass index. Eur $\mathrm{J}$ Clin Nutr 1994; 48:10S-27S.

5. Monteiro CA. Epidemiologia da obesidade. In: Halpern A, Matos AFG, Suplicy HL, Mancini MC, Zanella MT, organizadores. Obesidade. São Paulo: Lemos Editorial; 1998. p. 15-30.

6. Garrow JS. Indices of adiposity. Nutr Abstr Rev 1983; 53:697-708.

7. Norgan NG. Interpretation of low body mass indices: Australian Aborigines. Am J Physic Anthropol 1994; 94:229-37.

8. Rush EC, Plank LD, Laulu MS, Robinson SM. Prediction of percentage body fat from anthropometric measurements: comparison of New Zealand European and Polynesian young women. Am J Clin Nutr 1997; 66:2-7.

9. Eveleth PB, Tanner JM. Worldwide variation in human growth. 2nd Ed. Cambridge: Cambridge University Press; 1990.

10. Santos RV, Coimbra Jr. CEA. Socioeconomic differentiation and body morphology in the Suruí of Southwestern Amazonia. Curr Anthropol 1996; 37:851-6.

11. Leite MS, Gugelmin SA, Santos RV, Coimbra Jr. CEA. Perfis de saúde indígena, tendências nacionais e contextos locais: reflexões a partir do caso Xavánte, Mato Grosso. In: Coimbra Jr. CEA, Santos RV, Escobar AL, organizadores. Epidemiologia e saúde dos povos indígenas no Brasil. Rio de Janeiro: Editora Fiocruz/ABRASCO; 2003. p. 105-25.

12. Cardoso AM, Mattos IE, Koifman RJ. Prevalência de fatores de risco para doenças cardiovasculares na população Guaraní-Mbyá do Estado do Rio de Janeiro. Cad Saúde Pública 2001; 17:345-54.

13. Gugelmin SA, Santos RV. Ecologia humana e antropometria nutricional de adultos Xavánte, Mato Grosso, Brasil. Cad Saúde Pública 2001; 17:31322.

14. Capelli JCS, Koifman S. Avaliação do estado nutricional da comunidade indígena Parkatêjê, Bom Jesus do Tocantins, Pará, Brasil. Cad Saúde Pública $2001 ; 17: 433-7$.
15. Souza LG, Santos RV. Perfil demográfico da população indígena Xavánte de Sangradouro-Volta Grande, Mato Grosso (1993-1997), Brasil. Cad Saúde Pública 2001; 17:355-65.

16. Lohman TG, Roche AF, Martorell R. Anthropometric standardization reference manual. Champaign: Human Kinetics; 1988.

17. Heymsfield SB, McManus C, Smith J, Stevens V, Nixon DW. Anthropometric measurement of muscle mass: revised equations for calculating bonefree arm muscle area. Am J Clin Nutr 1982; 36: 680-90.

18. World Health Organization. Physical status: the use and interpretation of anthropometry. Geneva: World Health Organization; 1995. (Technical Report Series, 854).

19. Malina RM. Regional body composition: age, sex, and ethnic variation. In: Roche AF, Heymsfield SB, Lohman TG, editors. Human body composition. Champaign: Human Kinetics; 1996. p. 217 55.

20. Van Loan MD. Total body composition: birth to old age. In: Roche AF, Heymsfield SB, Lohman TG, editors. Human body composition. Champaign: Human Kinetics; 1996. p. 205-15.

21. World Health Organization. Diet, nutrition, and the prevention of chronic diseases. Geneva: World Health Organization; 1990. (WHO Technical Report Series, 797).

22. Coimbra Jr. CEA, Flowers NM, Salzano FM, Santos RV. The Xavante in transition: health, ecology and bioanthropology in Central Brazil. Ann Arbor: University of Michigan Press; 2002.

23. Neel JV, Salzano FM, Junqueira PC, Keiter F, Maybury-Lewis D. Studies on the Xavante Indians of the Brazilian Mato Grosso. Am J Hum Gen 1964; 16:52-140.

24. Santos RV. Crescimento físico e estado nutricional de populações indígenas brasileiras. Cad Saúde Pública 1993; 9:46-57.

25. Coimbra Jr. CEA, Santos RV. Saúde, minorias e desigualdade: algumas teias de inter-relações, com ênfase nos povos indígenas. Ciênc Saúde Coletiva 2000; 5:125-32.

26. Santos RV, Coimbra Jr. CEA. Cenários e tendências da saúde e da epidemiologia dos povos indígenas no Brasil. In: Coimbra Jr. CEA, Santos RV, Escobar AL, organizadores. Epidemiologia e saúde dos povos indígenas no Brasil. Rio de Janeiro: Editora Fiocruz/ABRASCO; 2003. p. 13-47.

27. Foucan L, Hanley J, Deloumeaux J, Suissa S. Body mass index (BMI) and waist circunference (WC) as screening tools for cardiovascular risk factors in Guadeloupean women. J Clin Epidemiol 2002; 55:990-6.

Recebido em 27/Jun/2005

Versão final reapresentada em 14/Dez/2005

Aprovado em 03/Jan/2006 